\title{
CORRESPONDENCE
}

\section{Humane care for vulnerable people}

The article on depot antipsychotic medication by Patel \& David (2005) is welcome, as is its accompanying invited commentary (Barnes, 2005). An issue hinted at but understated in both is that of the risk to others posed by some individuals with schizophrenia and related disorders. Although people with schizophrenia whose symptoms are well-controlled are no more a risk to others than is anyone in the general population, those with active psychosis do pose an elevated risk. The number of people with schizophrenia who kill others or who inflict serious violence on others when stabilised on depot, although not zero, is very low. A number of homicides by individuals with schizophrenia have followed discontinuation of depot and replacement by oral medication, with which full adherence has not occurred (Howlett, 1998). Even among in-patients, I have known individuals returned to intensive care units following discontinuation of depot in preference for oral medication, in both general psychiatric and forensic psychiatric units. In a few cases all that is holding back relapse into dangerous behaviour is the treatment for the mental illness, and some tragedies may be prevented by ensuring that the patient takes the treatment.

Patel \& David refer to the modern ethos of a partnership with the patient rather than paternalism. In my view a good father develops a partnership with his children but he still takes care to protect them from harm when they are as yet unable to do so themselves. Adult psychiatric patients are not children but they are vulnerable people often with impaired judgement. The contemporary adverse perspective on medical paternalism need not serve to discredit an historical medical tradition involving humane care.

Barnes, T. R. E. (2005) Why indeed? Invited commentary on: Why aren't depot antipsychotics prescribed more often and what can be done about it? Advances in Psychiatric Treatment, 11, 211-213.

Howlett, M. (1998) Medication, Non-Compliance and Mentally Disordered Offenders: The Role of Non-Compliance in Homicide by People with Mental Illness and Proposals for Future Policy. A Study of Independent Inquiry Reports. London: Zito Trust.

Patel, M. X. \& David, A. S. (2005) Why aren't depot antipsychotics prescribed more often and what can be done about it? Advances in Psychiatric Treatment, 11, 203-211.

Harvey Gordon Consultant Forensic Psychiatrist, Oxford Clinic Medium Secure Unit, and Honorary Senior Lecturer in Forensic Psychiatry, University of Oxford, UK. E-mail: Anna.Kennedy@ oxmhc-tr.nhs.uk

\section{Eating disorders - new interest}

Bob Palmer's (2006) article considering the advances (or lack of them) in the management of anorexia nervosa over the past 10 years comes at a time of renewed interest in the subject of eating disorders. This has been contributed to by NICE guidelines (National Collaborating Centre for Mental Health, 2004) and various initiatives and publications by the Royal College of Psychiatrists, including the 19982003 Changing Minds Campaign (http://www. rcpsych.ac.uk/campaigns/cminds/anorexia.htm), various information leaflets (http://www.rcpsych. ac.uk/info/eatdis.htm) and a document on guidelines for the nutritional management of anorexia nervosa (Royal College of Psychiatrists, 2005).

Eating disorders used to be associated with young women working in the beauty and fashion industries, in a culture that promotes a stereotyped concept of beauty and thinness. It is now accepted that they affect (mostly) young women and occasionally young men in all walks of life. Nevertheless, eating disorders tend to be neglected and underreported, with patients unwilling to seek help or engage in treatment. There is a great need for more public awareness of the illness and its consequences. It was therefore very pleasing to read that Paula Abdul, an award-winning performer and celebrity judge on the TV series American Pop Idol, has received the American National Eating Disorders Association's Profiles in Living Award for her work as an ambassador for the Association, having had her own battle with an eating disorder.

There is a lack of new substantial research on innovative treatments for eating disorders, but there have been some welcome developments in putting in place more resources for treatment. The Hampshire Partnership NHS Trust, for example, plans to set up a new eight-bedded eating disorder unit. I now hope for effective new treatments to offer patients who have eating disorders that will improve their care and provide a better outcome.

National Collaborating Centre for Mental Health (2004) Eating Disorders: Core Interventions in the Treatment and Management of Anorexia Nervosa, Bulimia Nervosa and Related Eating Disorders. National Clinical Practice Guideline No. CG9. Leicester \& London: British Psychological Society \& Gaskell.

Palmer, B. (2006) Come the revolution. Revisiting: The management of anorexia nervosa. Advances in Psychiatric Treatment, 12, 5-12.

Royal College of Psychiatrists (2005) Guidelines for the Nutritional Management of Anorexia Nervosa (Council Report CR130). London: Royal College of Psychiatrists.

Marlene M. Kelbrick Senior House Officer, Hampshire Partnership NHS Trust, Parklands Hospital, Basingstoke, UK. E-mail: kelbrickm@aol.com 